\title{
Nanoscale Investigation of Layered Oxychloride Intergrowth Photocatalysts for Visible Light Driven Water Splitting
}

Kaustav Chatterjee ${ }^{1}$, Sandra Bueno ${ }^{1}$, Sara Skrabalak ${ }^{1}$, Vinayak Dravid ${ }^{2}$ and Roberto dos Reis ${ }^{2}$

${ }^{1}$ Indiana University - Bloomington, Bloomington, Indiana, United States, ${ }^{2}$ Northwestern University, Evanston, Illinois, United States

Photocatalytic water splitting is a promising platform for clean and renewable energy generation from solar energy. Metal mixed anion compounds such as oxynitrides, oxysulphides, and oxyhalides have emerged as promising photocatalysts for water splitting, owing to their negative valence band maximum (VBM) compared to conventional oxides $[1,2]$. Substantial mixing of the p-orbitals from the non-oxide anions (e.g., N-2p, Cl-3p) with the O-2p orbitals cause a negative shift in the VBM. Unfortunately, most mixed-anion compounds undergo self-oxidation of non-oxide anions by photogenerated holes, thereby imposing surface modifications to overcome the oxidative photo-corrosion [3, 4]. Herein, we present the structural and compositional nanoscale characterization of new tantalum-gadolinium (Ta-Gd) based layered intergrowth perovskite oxychloride, $\mathrm{Bi} 4 \mathrm{TaO}_{8} \mathrm{Cl}-\mathrm{Bi}_{2} \mathrm{GdO}_{4} \mathrm{Cl}$, as a stable material under visible light for photocatalysis. It is revealed that the $\mathrm{VBMs}$ of $\mathrm{Bi} 4 \mathrm{TaO}_{8} \mathrm{Cl}$ and $\mathrm{Bi}_{2} \mathrm{GdO}_{4} \mathrm{Cl}$ consist mainly of O$2 \mathrm{p}$ orbitals, instead of Cl-3p, with a negative shift in the VBM [4]. Thus, they possess narrow bandgaps for visible light absorption and photogenerated holes populated at the O-2p orbitals will not lead to photooxidation but to oxidize water.

$\mathrm{Bi}_{4} \mathrm{TaO}_{8} \mathrm{Cl}$ is a Sillén-Aurivillius perovskite phase consisting of single-layer $\mathrm{TaO}_{4}$ perovskite blocks that are separated by $\left(\mathrm{Bi}_{2} \mathrm{O}_{2}\right)_{2} \mathrm{Cl}$ blocks, while $\mathrm{Bi}_{2} \mathrm{GdO}_{4} \mathrm{Cl}$ presents a $P 4 / \mathrm{mmm}$ structure. Two different projections, along the [110] and c-axes, of these structures are shown in Fig. 1. The new intergrowth materials were prepared by molten flux syntheses using halide fluxes to enhance reaction rates by increasing rate of diffusion, enhanced contact area between reactants and reduce halogen defects on the surface. We have systematically varied the Ta:Gd molar ratio in the intergrowths to rationalize the effect of charge separation and band structure changes towards photocatalytic water splitting.

In addition, we used aberration corrected scanning transmission electron microscopy (STEM) and energy dispersive X-Ray spectroscopy (EDX) to achieve a complete picture of the final structure and composition. Fig. 1(e) presents STEM-EDX maps accompanied with simultaneously acquired high-angle annular dark field (HAADF) taken from the sample with Ta:Gd molar ration of 1:1. It shows the nanoscale homogeneity within the acquired region with the expected molar Ta:Gd molar ratio, which suggests the formation of intergrowths.

HAADF and annular bright-field (ABF) images were taken from different zone axes to obtain local crystallographic information. Fig. 3(a) and 3(b) show, respectively, HAADF and ABF images taken along [100] pseudocubic axis. In the HAADF image, one can observe the projected A-sites, comprised by $\mathrm{Bi} / \mathrm{Cl}$, and the $\mathrm{B}$-sites, comprised by the intercalation between $\mathrm{Bi} / \mathrm{Ta} / \mathrm{Gd}$, whereas in the $\mathrm{ABF}, \mathrm{O}$-sites are highlighted. In HAADF the contrast is based on scattering amplitude whose sensitivity depends on the scattering power of the relevant atoms (so called Z-contrast images). Therefore, A-sites show lower intensity contrast due to the lower atomic number average $\left(\mathrm{Z}_{\mathrm{Bi}}=83, \mathrm{Z}_{\mathrm{Cl}}=17\right)$ in comparison to the $\mathrm{B}$-sites $\left(Z_{\mathrm{Bi}}=83, \mathrm{Z}_{\mathrm{Ta}}=73, \mathrm{Z}_{\mathrm{Gd}}=64\right)$. Fig. 3(c) and (d) shows, respectively, HAADF and ABF images from the same sample along [110] zone axis. Through this projection, one can observe two distinct layers in the $\mathrm{HAADF}$ image contrast, Bi-rich and $\mathrm{Ta} / \mathrm{Gd} / \mathrm{Cl}$ (pointed out by the arrows). 
The nanoscale structural and compositional investigations provide a better understanding for the high apparent quantum efficiency for the oxygen evolution reaction that is demonstrated when this photocatalyst is loaded with $1 \mathrm{wt} \% \mathrm{RuO}_{2}$ co-catalyst and irradiated with visible light. These results could provide new strategies for designing durable photocatalysts by tuning the electrostatic interaction between Gr-VA elements and O-2p orbitals [5].

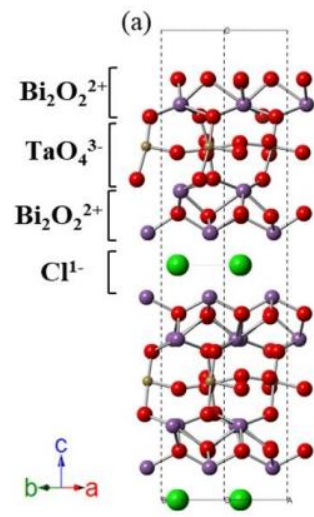

(c) (b)

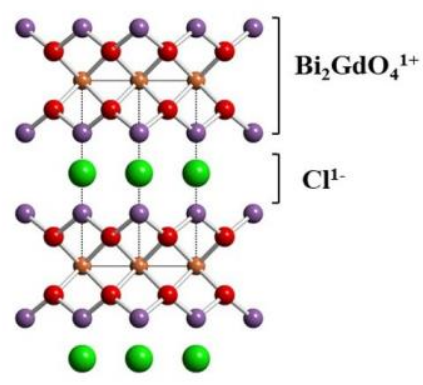

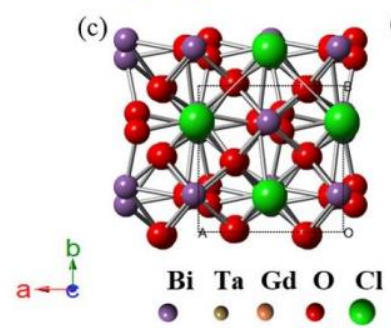

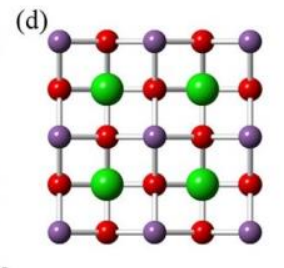

- $\odot \odot$

(e)
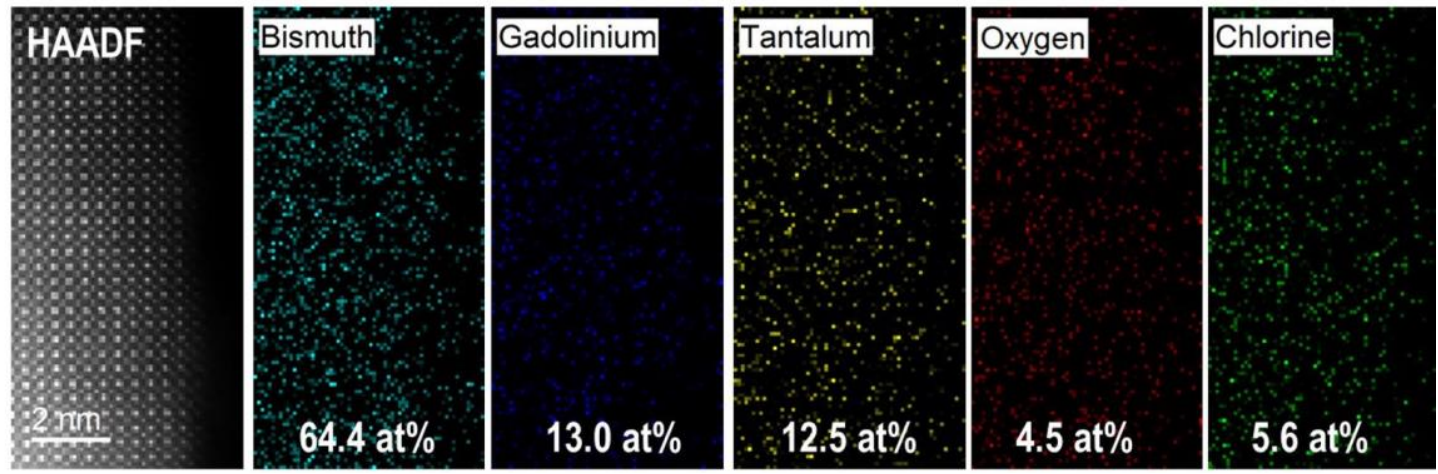

Figure 1. Crystal structures of (a, c) Sillén-Aurivillius layered perovskite-Bi4TaO8Cl (P21cn) and (b, d) $\mathrm{Bi} 2 \mathrm{GdO} 4 \mathrm{Cl}$ (P4/mmm) viewed along two projections [110] and [001]; (e) EDX maps from sample with Ta:Gd molar ratio of 1:1. Mapping shows the nanoscale homogeneity of the elements. 

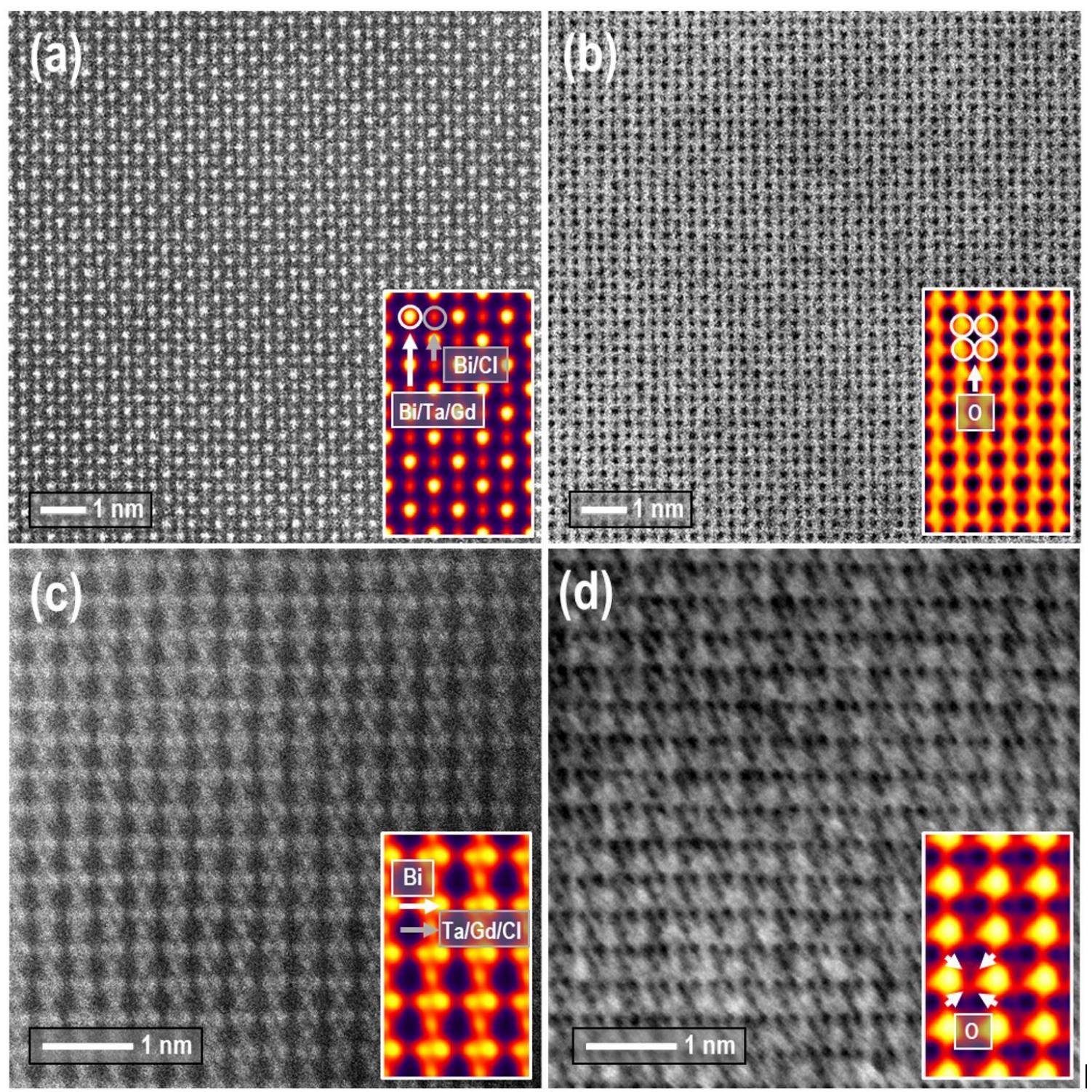

Figure 2. HAADF images from sample with Ta:Gd molar ratio of 1:1 viewed along two different zone axes. (a) and (b) are, respectively, ADF and ABF taken along [001] axis. Layered aspect of the Sillén-Aurivillius structure is depicted in (c) and (d) that are, respectively, ADF and ABF images taken along [110] axis. Mean unit cells averages are shown as inset to emphasize the intensity contrast in each of the micrographs. Arrows and labels point the most likely site atomic occupancy.

References

[1] Abeysinghe, D.; Skrabalak, S. E. Toward Shape-Controlled Metal Oxynitride and Nitride Particles for Solar Energy Applications. ACS Energy Lett. 2018, 3, 1331-1344.

[2] Li, J.; Yu, Y.; Zhang, L. Bismuth Oxyhalide Nanomaterials: Layered Structures Meet Photocatalysis. Nanoscale 2014, 6, 8473-8488.

[3] Fujito, H.; Kunioku, H.; Kato, D.; Suzuki, H.; Higashi, M.; Kageyama, H.; Abe, R. Layered Perovskite Oxychloride $\mathrm{Bi}_{4} \mathrm{NbO}_{8} \mathrm{Cl}$ : A Stable Visible Light Responsive Photocatalyst for Water Splitting. J. Am. Chem. Soc. 2016, 138, 2082-2085. 
[4] Kato, D.; Hongo, K.; Maezono, R.; Higashi, M.; Kunioku, H.; Yabuuchi, M.; Suzuki, H.; Okajima, H.; Zhong, C.; Nakano, K.; et al. Valence Band Engineering of Layered Bismuth Oxyhalides toward Stable Visible-Light Water Splitting: Madelung Site Potential Analysis. J. Am. Chem. Soc. 2017, 139, 18725-18731.

[5] This work was supported by MRSEC program (NSF DMR-1720139), made use of the EPIC facility of Northwestern University's NUANCE Center, which has received support from the Soft and Hybrid Nanotechnology Experimental (SHyNE) Resource (NSF ECCS-1542205); the MRSEC program (NSF DMR-1121262) at the Materials Research Center; the International Institute for Nanotechnology (IIN); the Keck Foundation; and the State of Illinois, through the IIN. Additional support to K.C., S.A.B., and S.E.S. come from Indiana University, with S.A.B. supported by NSF DGE-1342962 (Graduate Research Fellowship Program). 